\title{
Theoretical Study of Ag Interactions in Amorphous Silica RRAM Devices
}

\author{
K. Patel ${ }^{1,2}$, J. Cottom ${ }^{1}$, M. Bosman ${ }^{2,3}$, A. J. Kenyon ${ }^{4}$ and A. L. Shluger ${ }^{1}$ \\ ${ }^{1}$ Department of Physics and Astronomy, University College London, Gower Street, London, WC1E 6BT, UK. \\ ${ }^{2}$ Institute of Materials Research and Engineering, A*STAR (Agency for Science, Technology and Research), 2 Fusionopolis \\ Way, Singapore 138634, Singapore. \\ ${ }^{3}$ Department of Materials Science and Engineering, National University of Singapore, Singapore 117575, Singapore \\ ${ }^{4}$ Department of Electrical \& Electronic Engineering, University College London, Torrington Place, London, WC1E 7JE, UK.
}

In this study, Density Functional Theory (DFT) calculations were used to model the incorporation and diffusion of $\mathrm{Ag}$ in $\mathrm{Ag} / \mathrm{a}-\mathrm{SiO}_{2} / \mathrm{Pt}$ resistive random-access memory (RRAM) devices. The Ag clustering mechanism is vital for understanding device operation and at this stage is unknown. In this paper an $O$ vacancy $\left(V_{0}\right)$ mediated cluster model is presented, where the $V_{0}$ is identified as the principle site for $\mathrm{Ag}^{+}$reduction. The $\mathrm{Ag}^{+}$interstitial is energetically favored at the Fermi energies of $\mathrm{Ag}$ and $\mathrm{Pt}$, indicating that $\mathrm{Ag}^{+}$ions are not reduced at the Pt electrode via electron tunneling. Instead, $\mathrm{Ag}^{+}$ions bind to $\mathrm{V}_{\mathrm{o}}$ forming the $\left[\mathrm{Ag} / \mathrm{Vo}_{\mathrm{o}}\right]^{+}$complex, reducing $\mathrm{Ag}^{+}$via charge transfer from the $\mathrm{Si}$ atoms in the vacancy. The $[\mathrm{Ag} / \mathrm{Vo}]^{+}$complex is then able to trap an electron forming $[\mathrm{Ag} / \mathrm{Vo}]^{0}$ at the Fermi energy of Pt. This complex is then able to act as a nucleation site for of $\mathrm{Ag}$ clustering with the formation of $\left[\mathrm{Ag}_{2} / \mathrm{Vo}\right]^{+}$which is reduced by the above mechanism.

\section{INTRODUCTION}

In recent years, considerable effort has been devoted to the development of RRAM devices. High switching speeds, endurance and scalability combined with low power consumption make RRAM a viable alternative to FLASH memory [1]. RRAM devices operate via the resistive switching phenomenon, where the generation and dissolution of electron transport modes across a dielectric layer determine its resistance between high and low states. Typically, before this switching is observed, a conductive filament is formed across the dielectric layer in a process analogous to soft dielectric breakdown.

The nature of the conductive path varies with material and microstructure. Devices containing an oxide layer, typically amorphous $\mathrm{SiO}_{2}\left(\mathrm{a}-\mathrm{SiO}_{2}\right)$, have been shown to operate under two distinct mechanisms; the electrochemical metallization mechanism (ECM) and the valence change mechanism (VCM) [2], [3]. As such, understanding how these mechanisms interact is important to the operation and optimization of RRAM devices. It is generally understood for ECM memories that metal ions migrate through the dielectric layer to the inert electrode via field-assisted diffusion. Metallic clusters then form, creating a conducting path across the dielectric. The specific oxidation and reduction processes remain unclear and are the subject of vigorous debate. An atomistic understanding of these processes is vital before the nucleation and propagation of metal clusters can be understood and optimized.

$\mathrm{Ag} / \mathrm{a}-\mathrm{SiO}_{2} / \mathrm{Pt}$ devices have been studied extensively experimentally thus providing a wealth of experimental data for comparison with theoretical calculations [3]. The $\mathrm{Ag}_{\mathrm{i}}$ and $\mathrm{V}_{\mathrm{O}^{-}}$ mediated mechanisms for $\mathrm{Ag}$ reduction and cluster nucleation are investigated in this work.

\section{METHODOLOGY}

The a- $\mathrm{SiO}_{2}$ models were generated via molecular dynamics melt-quench using the ReaxFF [4] force field as implemented in the LAMMPS code [5]. The initial structure was a 216 atom supercell of cubic $\beta$-cristobalite $\left(\mathrm{SiO}_{2}\right)$. To ensure sufficient sampling of the amorphous structures was achieved, 30 models were generated. In each case the system was first equilibrated at $300 \mathrm{~K}$ and a pressure of $1 \mathrm{~atm}$. The pressure was fixed at $1 \mathrm{~atm}$ whilst the temperature was linearly ramped to $5000 \mathrm{~K}$ using a Berendsen thermostat [6]. The temperature was maintained at $5000 \mathrm{~K}$ for $40 \mathrm{ps}$ to ensure the initial structure had been completely melted then cooled to $0 \mathrm{~K}$ at a rate of $8 \mathrm{~K} \mathrm{ps}^{-1}$. The final structures were then optimized using DFT with the PBE0-TC_LRC functional [7] in the CP2K code [8]. The densities (mean 2.20 $\mathrm{g} / \mathrm{cm}^{3}$ ), distributions of $\mathrm{Si}-\mathrm{O}$ bonds and $\mathrm{Si}-\mathrm{O}-\mathrm{Si}$ angles, and neutron structure factors of the models were consistent with those obtained in previous studies [9], [10].

DFT calculations were carried out using the Gaussian Plane Wave method (GPW) [11] in the CP2K code. A plane wave cutoff and relative cut-off of $600 \mathrm{Ry}$ and $40 \mathrm{Ry}$ were used respectively, with double zeta valence polarized (DZVP) molecularly optimized (MOLOPT) basis sets [12] and GoedeckerTeter-Hutter (GTH) pseudopotentials [13]. The PBE0-TC-LRC functional truncated at $2 \AA$ was used with the auxiliary density matrix method (ADMM) implementation to reduce computational cost [14].

To study the Ag interstitial and its charge states in a- $\mathrm{SiO}_{2}$, the complete sampling of one supercell was carried out for the charge states $-1 \leq \mathrm{q} \leq+2$. Out of the 30 produced models, the supercell with the density closest to the mean density was selected. A 3dimensional grid with a $2 \AA$ spacing was generated across the supercell giving 512 grid points. From these, grid points were excluded if there was a silica atom less than $1.34 \AA$ away, corresponding to the atomic radius of $\mathrm{Ag}$ [15]. The remaining 259 points were used as initial Ag interstitial site locations. In separate simulations, Ag was added at each point and the geometry was optimized using the PBE functional. Unique sites were determined as those having any atom in the cell displaced by more than $0.2 \AA$, an energy difference of greater than $0.1 \mathrm{eV}$ or a Mulliken charge difference of greater than $0.05|e|$. The unique sites were then optimized using the PBE0-TC-LRC functional.

The $\mathrm{O}$ vacancy was modelled at 37 sites in a periodic cell chosen using a ring sampling method, where each $\mathrm{O}$ in an $\mathrm{N}$-membered ring was removed, and the geometry optimized $(\mathrm{N}=3 \leq \mathrm{N} \leq 9)$. 
The defect formation energy, $E_{\text {form }}$, is calculated using the standard formalism of Northrup and Zhang [16]:

$$
E_{\text {form }}=E_{\text {defect }}-E_{\text {pristine }}-\mu_{x}+q\left(\Delta E_{F}+E_{v}\right)+E_{\text {corr }},
$$

where $E_{\text {defect }}$ is the total energy of the system with the defect, $E_{\text {pristine }}$ is the total energy of the pristine system, $\mu_{\mathrm{x}}$ is the chemical potential of chemical species, $q$ is the charge of the system, $\Delta E_{F}$ is the Fermi level position, $E_{v}$ is the potential alignment and $E_{c o r r}$ is the charge correction. The Lany-Zunger finite size charge correction scheme was utilized to correct the formation energies of the charges defects [17]. The chemical potential of $\mathrm{O}, \mu_{O}$, is calculated as one half the energy of an $\mathrm{O}_{2}$ molecule. The chemical potential of $\mathrm{Ag}, \mu_{\mathrm{Ag}}$, is calculated from a 162-atom cell of $\mathrm{Ag}_{2} \mathrm{O}$ as:

$$
\mu_{A g}=\frac{1}{2 n}\left(E_{\mathrm{Ag}_{2} \mathrm{O}}-\frac{1}{2} n \mu_{\mathrm{O}_{2}}\right),
$$

where $n$ is the number of $\mathrm{Ag}_{2} \mathrm{O}$ units, $\mathrm{E}_{\mathrm{Ag} 2 \mathrm{O}}$ is the total energy of the $\mathrm{Ag}_{2} \mathrm{O}$ system and $\mu_{\mathrm{O} 2}$ is the chemical potential of an $\mathrm{O}$ molecule. The Fermi energy positions of $\mathrm{Ag}$ and $\mathrm{Pt}$ have been experimentally measured at $4.96 \mathrm{eV}$ and $4.11 \mathrm{eV}$ with respect to the a- $\mathrm{SiO}_{2}$ valence band respectively [18]-[20].

\section{RESULTS AND DISCUSSION}

To understand the redox processes involved in Ag migration and clustering, it is first important to find the incorporation energy of $\mathrm{Ag}$ as a function of Fermi energy. From this, the charge state of $\mathrm{Ag}$ can be determined at the $\mathrm{Ag}$ and $\mathrm{Pt}$ electrodes under bias. Furthermore, studying the unique sites for Ag can gleam information about the accessible areas in $\mathrm{a}-\mathrm{SiO}_{2}$ during migration.

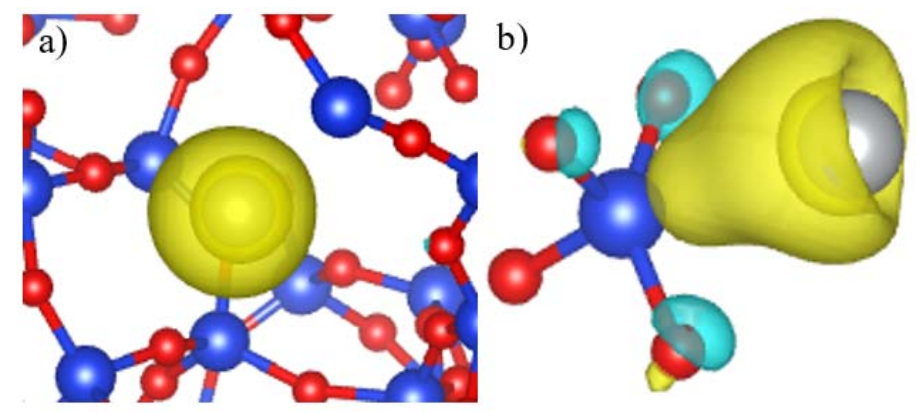

Fig. 1 Schematic showing the HOMO state of two configurations of the $\mathrm{Ag}^{0}$ interstitial in a-SiO ${ }_{2}$. The silver, blue and red spheres correspond to $\mathrm{Ag}, \mathrm{Si}$ and $\mathrm{O}$ atoms respectively. The positive and negative charge density is shown as blue and yellow iso-surfaces respectively at a value of \pm 0.07 . a) $\mathrm{Ag}^{0}$ occupies a void in the lattice, with the HOMO state mostly $\mathrm{Ag} \mathrm{s}$ in character. b) $\mathrm{Ag}^{0}$ resides near a wide $\mathrm{O}-\mathrm{Si}-\mathrm{O}$ angle bond, with charge donation from $\mathrm{Ag}$ to $\mathrm{Si}$

In 18 out of the 34 unique sites found (Fig. 1a) $\mathrm{Ag}^{0}$ is located in a void, where the highest occupied molecular orbital (HOMO) is primarily Ag s-character. In this case the mean Mulliken charge of $\mathrm{Ag}$ is $0.02|e|$. The remaining 16 sites (Fig. 1b) involve the widening of a proximate $\mathrm{O}-\mathrm{Si}-\mathrm{O}$ bond angle and the donation of charge from Ag to Si. Mulliken charge analysis gives a mean Ag charge of $0.25|e|$ and with a mean charge of $0.19|e|$ donated to the $\mathrm{Si}$. Previous studies have shown that O-Si-O bond angles $\geq 130^{\circ}$ are precursor sites for electron trapping [21]. Precursor sites can trap two electrons causing the weakening and stretching of an $\mathrm{O}$ Si bond by $0.5 \AA$. This in turn results in lower barriers $(\sim 1 \mathrm{eV})$ for
$\mathrm{V}_{\mathrm{O}}$ formation [22]. In this study 13 of the 34 unique sites resulted in the sponaneous widening of an O-Si-O bond angle $\geq 130^{\circ}$.

The mean, minimum, and maximum incorporation energies of $\mathrm{Ag}^{0}$ are $3.1 \mathrm{eV}, 2.8 \mathrm{eV}$, and $6.1 \mathrm{eV}$ respectively. The incorpoation energy of $\mathrm{Ag}^{0}$ was found to be strongly correlated to the local steric environment. The coordination number (defined as the number of atoms within $3 \AA$ of $\mathrm{Ag}^{0}$ ) increases with the incorporation energy whilst the Mulliken charge on the $\mathrm{Ag}$ decreases. The $\mathrm{Ag}^{0}$ atoms interacting with a widened $\mathrm{O}-\mathrm{Si}-\mathrm{O}$ bond angles have lower incorporation energies $(\sim 0.4 \mathrm{eV})$ than the void type defects with the same coordination number, suggesting the energetic cost of creating the wide angle O-Si-O is compensated by its interaction with $\mathrm{Ag}$.

Analysis of the 50 unique $\mathrm{Ag}^{+}$ion sites shows that $\mathrm{Ag}^{+}$ maintains a nearest neighbour distance of between 2.2 and $2.6 \AA$ to an $\mathrm{O}$ in the lattice. This differs from $\mathrm{Ag}^{0}$ where $18 \%$ of sites are in voids greater than $3 \AA$ from their nearest neighbour. The mean Mulliken charge of $\mathrm{Ag}$ in the +1 state is $0.78|e|$, with a strong correlation to the crowding of the lattice around the ion. As with $\mathrm{Ag}^{0}$, an increased coordination number yields a higher incorporation energy and a lower Mulliken charge ( $\min / \max \sim 0.4$ $|e|)$.

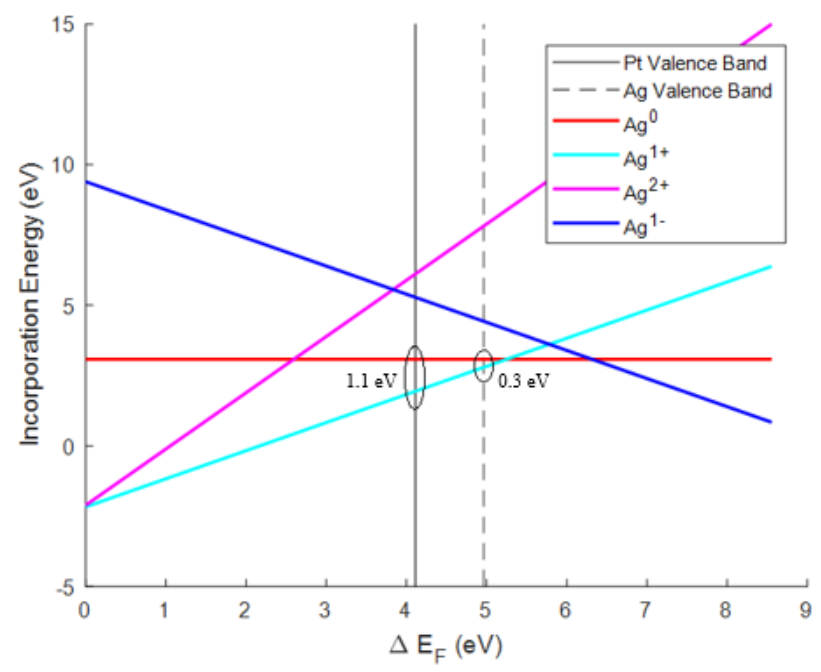

Fig. 2 Formation energy diagram of $259 \mathrm{Ag}$ interstitial sites in a-SiO2

Fig. 2 shows the defect formation energy of $\mathrm{Ag}$ in $\mathrm{a}-\mathrm{SiO}_{2}$. The Fermi position of $\mathrm{Ag}$ relative to $\mathrm{a}-\mathrm{SiO}_{2}$ suggests that the mean defect formation energy of $\mathrm{Ag}^{+}$is $0.3 \mathrm{eV}$ lower than $\mathrm{Ag}^{0}$ at the $\mathrm{Ag}$ electrode under equilibrium with the mean $(+1 / 0)$ transition at 5.25 $\mathrm{eV}$. This is problematic in understanding RRAM operation as devices operate under bias with forming voltages as high as +10 $\mathrm{V}$ and switching voltages $<+5 \mathrm{~V}$. Under these conditions, the Fermi energy position of $\mathrm{Ag}$ would shift towards the silica conduction band. In this case, $\mathrm{Ag}^{0}$ would be favored over $\mathrm{Ag}^{+}$ meaning an alternative interaction must be involved in the $\mathrm{Ag}^{+}$ion generation process, with previous experiments suggesting the presence of water in devices playing a crucial role in ion generation [23], [24]. The switching mechanism in ECM memories is usually bi-polar with negative bias applied to reset the device. In this case, further calculations are required to study the 
stability and charge transition levels of larger Ag clusters under operation.

Furthermore, from Fig. 2 we see that the defect formation energy of $\mathrm{Ag}^{+}$is $1.1 \mathrm{eV}$ lower than that of $\mathrm{Ag}^{0}$ at the Pt Fermi energy. This suggests that the reduction of $\mathrm{Ag}^{+}$ions by the tunneling of electrons from the Pt electrode is not energetically favorable. In this situation, $\mathrm{Ag}^{+}$ions are not reduced and clustering will not occur due to electrostatic repulsion between the charges. As such, the interaction of Ag with other defects in these devices is vital for understanding the redox processes. A plausible starting point is the $\mathrm{V}_{\mathrm{O}}$ as the high bias applied across the dielectric may assist in $\mathrm{V}_{\mathrm{O}}$ generation as seen in VCM devices.
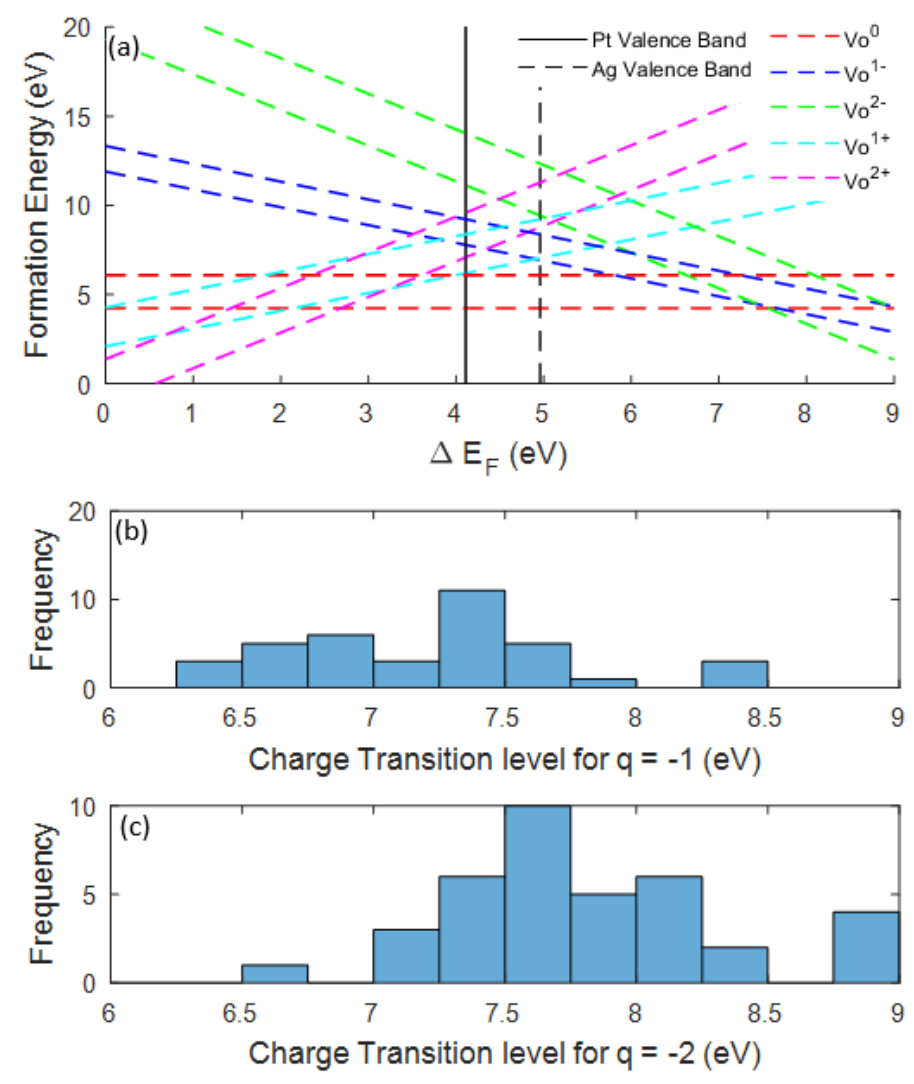

Fig. 3 a) Formation energies of $37 \mathrm{O}$ vacancies as a function of Fermi energy, $\Delta \mathrm{EF}$, where $\Delta \mathrm{F}=0$ at the $\mathrm{VBM}$. The dashed lines represent maxima and minima in the data distribution. $\mathrm{b})$ a histogram showing the $(0 /-1)$ charge transition position $\mathrm{c})$ a histogram showing the $(-1 /-2)$ charge transition position.

Analysis of the $37 \mathrm{O}$ vacancies give mean, minimum and maximum $\mathrm{V}_{\mathrm{O}}$ formation energies of $5.3 \mathrm{eV}, 4.2 \mathrm{eV}$ and $6.1 \mathrm{eV}$; single and double electron trapping become favorable at $\mathrm{E}_{\mathrm{F}}>7.2$ $\mathrm{eV}$ and $7.8 \mathrm{eV}$ respectively suggesting that electron trapping may happen at $3 \mathrm{~V}$. The calculations show a negative correlation of the $(0 /-1)$ and $(-1 /-2)$ transitions to the $\mathrm{Si}-\mathrm{Si}$ bond length, suggesting that vacancies with short $\mathrm{Si}-\mathrm{Si}$ bond lengths are energetically unfavorable to trap electrons. Fig. 4 compares the sampling method used in this study to the complete sampling of an a- $\mathrm{SiO}_{2}$ cell, showing the data in this study is skewed due to the higher number of $2.3 \AA$ to $2.4 \AA$ bond lengths sampled. Correcting for this would yield a lower mean defect formation energy and also reduce the Fermi energy of the $(0 /-1)$ and $(-1 /-2)$ transitions.

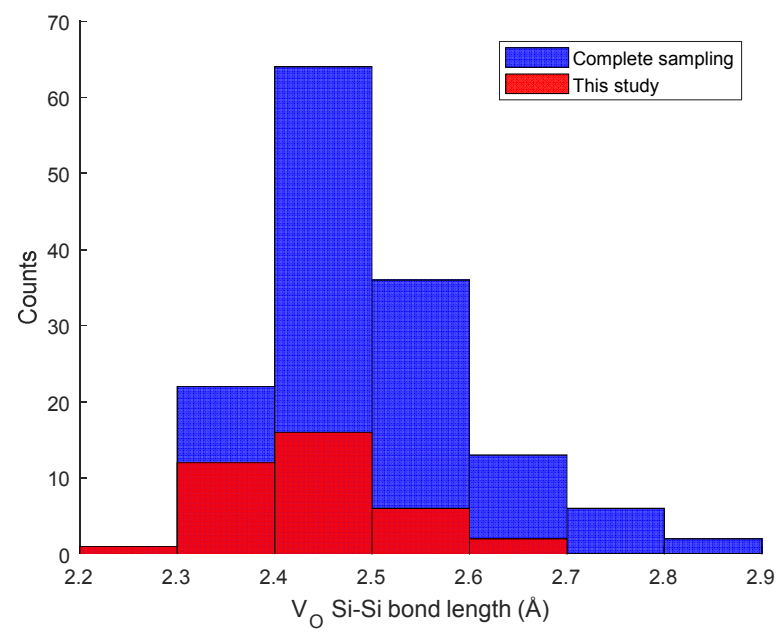

Fig 4. Histogram showing the Si-Si bond lengths for the $\mathrm{V}_{\mathrm{O}}$ sampling method used in this study compared to a complete sampling method, where every $\mathrm{O}$ is removed from the cell [25].

As the neutral vacancy $\left(\mathrm{V}_{\mathrm{O}}{ }^{0}\right)$ is favored at the Fermi energy of both $\mathrm{Ag}$ and $\mathrm{Pt}$, its interaction with $\mathrm{Ag}^{+}$is studied. For each of the 37 vacancies sampled, $\mathrm{a} \mathrm{Ag}^{+}$ion was placed in between the contributing Si atoms and the geometry was relaxed. In 27 of the 37 sampled sites the ion interacts with the vacancy to form a $[\mathrm{Ag} / \mathrm{Vo}]^{+}$complex. The mean Mulliken charge for $\mathrm{Ag}$ in the [Ag/Vo] $]^{+}$defect (Fig. 5a) is $0.17|e|$ compared to $0.82|e|$ for an $\mathrm{Ag}^{+1}$ interstitial. In this case, charge is donated from the $\mathrm{Si}$ atoms involved in the vacancy to $\mathrm{Ag}$, showing the $\mathrm{V}_{\mathrm{O}}$ to be a reduction site for $\mathrm{Ag}^{+}$ions. $\mathrm{Ag}$ can be further reduced by nearby oxygens to give a Mulliken charge of $0.05|e|$ (Fig. 5b) again highlighting the importance of local geometry on the Ag charge state. a)

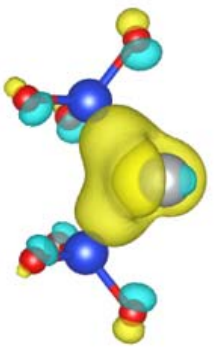

b)

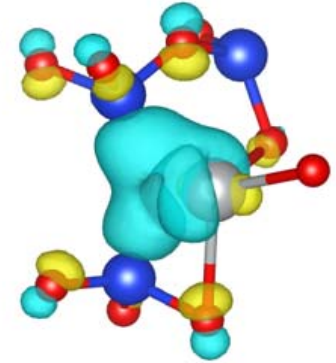

Fig. 5 Schematic showing the HOMO state of two configurations of the $\left[\mathrm{Ag} / \mathrm{V}_{\mathrm{o}}\right]^{+}$ defect. The silver, blue and red spheres correspond to $\mathrm{Ag}, \mathrm{Si}$ and $\mathrm{O}$ atoms respectively. The positive and negative charge density is shown as blue and yellow iso-surfaces respectively at a value of \pm 0.07 . Two configurations of the, a) typical $\left[\mathrm{Ag} / \mathrm{V}_{\mathrm{o}}\right]^{+}$defect, b) $\left[\mathrm{Ag} / \mathrm{V}_{\mathrm{o}}\right]^{+}$defect with nearby oxygens.

Each of the 10 sites that did not form a $[\mathrm{Ag} / \mathrm{Vo}]^{+}$complex involved an $\mathrm{V}_{\mathrm{O}}$ with a Si-Si bond length less than $2.4 \AA$, suggesting that the percentage of $\mathrm{Ag}^{+}$ions reduced by $\mathrm{V}_{\mathrm{O}}$ will be higher than the $72 \%$ found with the sampling method used in this study. Fig. 6 shows the $(+1 / 0)$ transition energy of the 37 vacancy sites sampled. In 9 of the 37 cases the neutral state was not favoured at any Fermi energy, with the $(+1 /-1)$ transitions marked with a star. For each of the complexes, a binding energy was calculated by removing the Ag from the vacancy and placing it in the lowest energy interstital site away from the vacancy. A postive binding energy was found for 10 of the 27 sites with a mean 
binding energy of $0.48 \mathrm{eV}$, each of which may serve as a $\mathrm{Ag}$ cluster nucleation site.

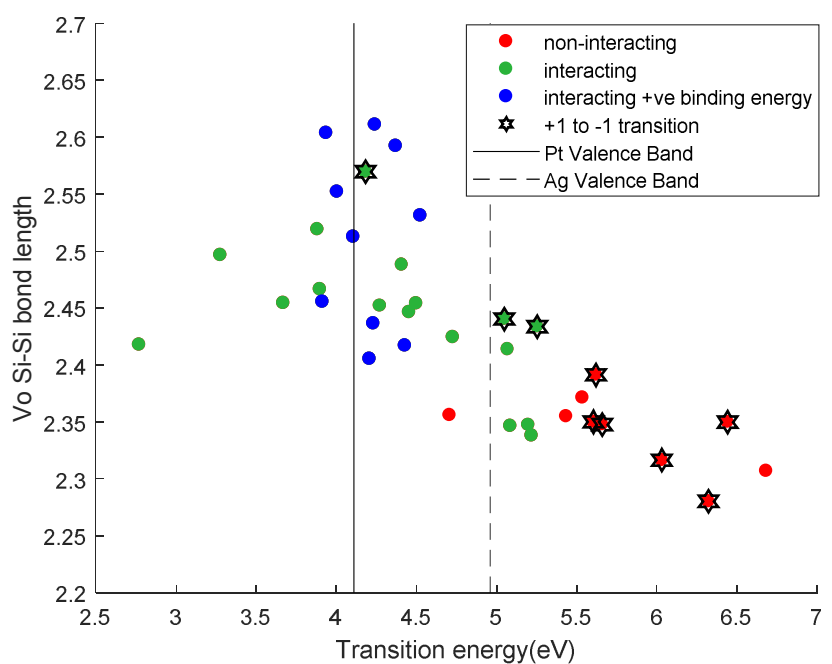

Fig. 6 Scatter diagram showing the $+1 / 0$ (dots) and $+1 /-1$ (stars) charge transitions and its correlation to the $\mathrm{Si}-\mathrm{Si}$ bond length. Red dots represent sites where $\mathrm{Ag}^{+}$ does not bind to $\mathrm{V}_{\mathrm{O}}$, green dots represent sites where $\mathrm{Ag}^{+}$is reduced by $\mathrm{V}_{\mathrm{O}}$ and blue dots represent sites where $\mathrm{Ag}^{+}$is reduced by $\mathrm{V}_{\mathrm{O}}$ and has a positive binding energy.

The neutral charge state of $\left[\mathrm{Ag} / \mathrm{V}_{\mathrm{O}}\right]^{0}$ is energetically favored at the Fermi energy of $\mathrm{Pt}$ for 9 of the 37 sites sampled. This suggests that $\mathrm{Ag}^{+}$ions will interact with $\mathrm{V}_{\mathrm{O}}$ to form $\left[\mathrm{Ag} / \mathrm{V}_{\mathrm{O}}\right]^{+}$complexes, which then trap an electron to form $\left[\mathrm{Ag} / \mathrm{V}_{\mathrm{O}}\right]^{0}$ near the Pt electrode.

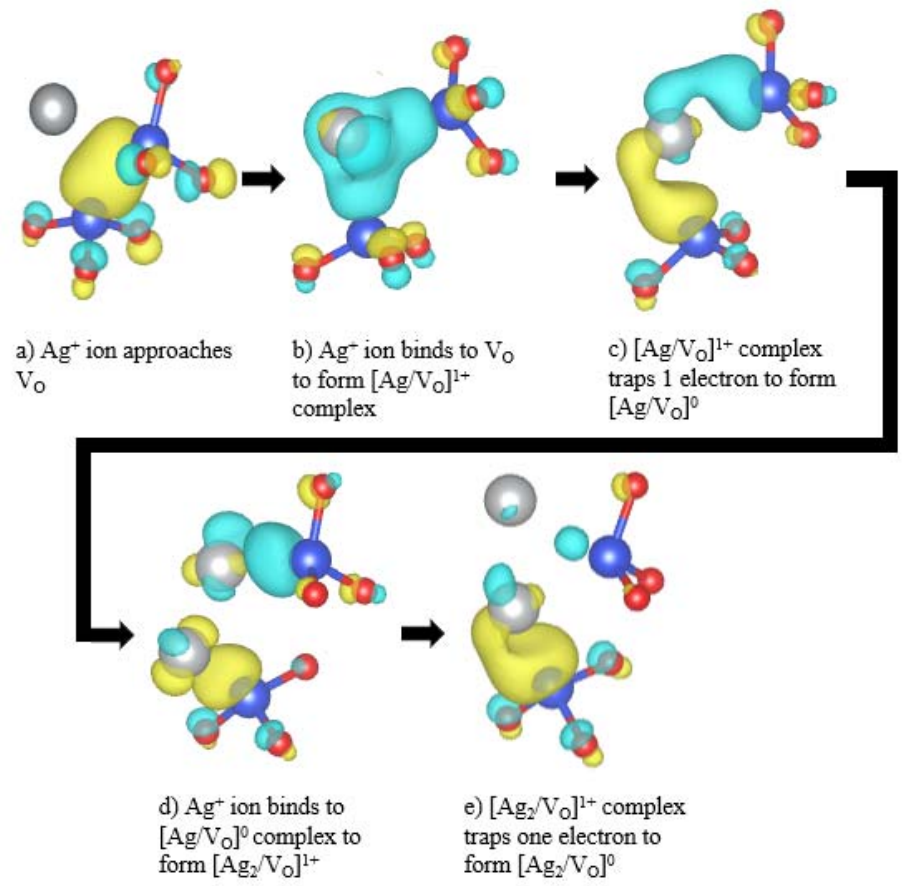

Fig. 7 Schematic showing the $\mathrm{V}_{\mathrm{O}}$ as a $\mathrm{Ag}^{+}$reduction site and subsequent cluster nucleation site. The silver, blue and red spheres correspond to $\mathrm{Ag}$, $\mathrm{Si}$ and $\mathrm{O}$ atoms respectively. The positive and negative charge density is shown as blue and yellow iso-surfaces respectively at a value of \pm 0.07 .

To study the $\mathrm{V}_{\mathrm{O}}$ as a cluster nucleation site (Fig. 7), a $\left[\mathrm{Ag} / \mathrm{V}_{\mathrm{O}}\right]$ complex with a $(+1 / 0)$ transition below the Pt Fermi energy was selected, and a second $\mathrm{Ag}$ ion was added to the system. The binding energy of the first $\mathrm{Ag}^{+}$to the $\mathrm{V}_{\mathrm{O}}$ in this case is $0.6 \mathrm{eV}$ (Fig.7 b). The $\left[\mathrm{Ag} / \mathrm{V}_{\mathrm{O}}\right]^{+}$is then favored to trap an electron with the $(+1 / 0)$ transition at a Fermi energy of $3.9 \mathrm{eV}$ compared to $\mathrm{Pt}$ at $4.11 \mathrm{eV}$ (Fig. 7c). Calculations for the additional $\mathrm{Ag}^{+}$ion in the $\left[\mathrm{Ag}_{2} / \mathrm{V}_{\mathrm{O}}\right]^{+}$state compared to occupying an interstitial site away from the vacancy give a binding energy of $0.6 \mathrm{eV}$ for $\mathrm{Ag}^{+}$to $\left[\mathrm{Ag} / \mathrm{V}_{\mathrm{O}}\right]($ Fig. $7 \mathrm{~d})$. The $(+1 / 0)$ transition of the $\left[\mathrm{Ag}_{2} / \mathrm{V}_{\mathrm{O}}\right]$ complex was found to be at $3.6 \mathrm{eV}$, showing that the $\left[\mathrm{Ag}_{2} / \mathrm{V}_{\mathrm{O}}\right]^{+}$complex would then be favored to trap an electron from the Pt electrode to form $\left[\mathrm{Ag}_{2} / \mathrm{V}_{\mathrm{O}}\right]^{0}$ (Fig. 7e). Though a more complete sampling of the $\mathrm{Ag}_{\mathrm{i}} / \mathrm{V}_{\mathrm{O}}$ interaction is required, it is clear $\mathrm{V}_{\mathrm{O}}$ plays a crucial role in clustering.

\section{CONCLUSION}

Two configurations of the $\mathrm{Ag}^{0}$ interstitial were found. In the first, $\mathrm{Ag}^{0}$ occupies a void in the lattice, with the HOMO consisting primarily of Ag s-character. The second involves the widening of an $\mathrm{O}-\mathrm{Si}-\mathrm{O}$ bond angle, and a subsequent charge donation from $\mathrm{Ag}$ to $\mathrm{Si}$. The $\mathrm{Ag}^{+}$interstitial was found to maintain a nearest neighbor distance of $2.2-2.6 \AA$ to an $\mathrm{O}$ in the lattice. The correlation of incorporation energy to the steric crowing around $\mathrm{Ag}$ by the lattice suggests that $\mathrm{Ag}$ will traverse $\mathrm{a}-\mathrm{SiO}_{2}$ via a void crossing mechanism.

The $\mathrm{Ag}^{+}$interstitial defect was found to have the lowest defect formation energy at the Fermi energies of $\mathrm{Ag}$ and $\mathrm{Pt}$, though the proximity of the $(+1 / 0)$ transition to the Ag Fermi energy suggests $\mathrm{Ag}^{0}$ will be favored under bias. The $\mathrm{Ag}^{+}$interstitial is favored at the Pt Fermi energy, suggesting $\mathrm{Ag}^{+}$ions are not reduced at the $\mathrm{Pt}$ electrodes via electron tunneling. Instead, $\mathrm{Ag}^{+}$ions are reduced by $\mathrm{O}$ vacancies after forming $\left[\mathrm{Ag} / \mathrm{V}_{\mathrm{O}}\right]^{+} .\left[\mathrm{Ag} / \mathrm{V}_{\mathrm{O}}\right]^{+}$are then able to trap an electron to form an $\left[\mathrm{Ag} / \mathrm{V}_{\mathrm{O}}\right]^{0}$. Due to biases introduced in the sampling method, it can be assumed that the rate of $\mathrm{Ag}^{+}$reduction is higher than calculated in this study. $\mathrm{A}$ second $\mathrm{Ag}^{+}$ion can bind to $\left[\mathrm{Ag} / \mathrm{V}_{\mathrm{O}}\right]^{0}$ to form $\left[\mathrm{Ag}_{2} / \mathrm{V}_{\mathrm{O}}\right]^{+}$, which is again favored to trap an electron giving a $\mathrm{Ag}_{2}$ dimer bound to a $\mathrm{V}_{\mathrm{O}}\left[\mathrm{Ag}_{2} / \mathrm{V}_{\mathrm{O}}\right]^{0}$, suggesting the $\mathrm{V}_{\mathrm{O}}$ site is a strong candidate for Ag cluster nucleation.

\section{ACKNOWLEDGEMENTS}

The A*STAR Graduate Academy is acknowledged for its graduate scholarship under the ARAP program. We also acknowledge funding provided by EPSRC (EP/L015862/1) and the use of the ARCHER High Performance Computing Facility via membership to the UK's HPC Materials Chemistry Consortium which is funded by EPSRC (EP/L000202).

\section{REFERENCES}

[1] T.-C. Chang, K.-C. Chang, T.-M. Tsai, T.-J. Chu, and S. M. Sze "Resistance random access memory," Mater. Today, vol. 19, no. 5, pp. 254-264, 2016.

[2] A. Mehonic et al., "Electrically tailored resistance switching in silicon oxide," Nanotechnology, vol. 23, no. 45, p. 455201, 2012.

[3] Y. Yang, P. Gao, S. Gaba, T. Chang, X. Pan, and W. Lu, "Observation of conducting filament growth in nanoscale resistive memories," Nat. Commun., vol. 3, p. 732, 2012.

[4] A. C. T. van Duin, A. Strachan, S. Stewman, Q. Zhang, X. Xu, and W. A. Goddard, "ReaxFFSiO Reactive Force Field for Silicon and Silicon Oxide Systems," J. Phys. Chem. A, vol. 107, no. 19, pp. 3803-3811, 
2003.

[5] S. Plimpton, "Fast Parallel Algorithms for Short-Range Molecular Dynamics," J. Comput. Phys., vol. 117, no. 1, pp. 1-19, 1995.

[6] H. J. C. Berendsen, J. P. M. Postma, W. F. van Gunsteren, A. DiNola, and J. R. Haak, "Molecular dynamics with coupling to an external bath," J. Chem. Phys., vol. 81, no. 8, pp. 3684-3690, 1984.

[7] M. Guidon, J. Hutter, and J. VandeVondele, "Robust Periodic Hartree-Fock Exchange for Large-Scale Simulations Using Gaussian Basis Sets," J. Chem. Theory Comput., vol. 5, no. 11, pp. 3010-3021, 2009.

[8] J. Vandevondele, M. Krack, F. Mohamed, M. Parrinello, T. Chassaing, and J. Hutter, "Quickstep: Fast and accurate density functional calculations using a mixed Gaussian and plane waves approach," Comput. Phys. Commun., vol. 167, no. 2, pp. 103-128, 2005.

[9] J. Sarnthein, A. Pasquarello, and R. Car, "Structural and Electronic Properties of Liquid and Amorphous $\mathrm{SiO}_{2}$ : $\mathrm{An} \mathrm{Ab}$ Initio Molecular Dynamics Study," Phys. Rev. Lett., vol. 74, no. 23, pp. 4682-4685, Jun. 1995.

[10] S. Susman et al., "Intermediate-range order in permanently densified vitreous $\mathrm{SiO}_{2}$ : A neutron-diffraction and molecular-dynamics study,' Phys. Rev. B, vol. 43, no. 1, pp. 1194-1197, Jan. 1991.

[11] B. G. LIPPERT, J. HUTTER, and M. PARRINELlO, "A hybrid Gaussian and plane wave density functional scheme," Mol. Phys., vol. 92, no. 3, pp. 477-488, 1997

[12] J. VandeVondele and J. Hutter, "Gaussian basis sets for accurate calculations on molecular systems in gas and condensed phases," $J$. Chem. Phys., vol. 127, no. 11, p. 114105, 2007.

[13] S. Goedecker, M. Teter, and J. Hutter, "Separable dual-space Gaussian pseudopotentials,” Phys. Rev. B, vol. 54, no. 3, pp. 1703-1710, Jul. 1996.

[14] M. Guidon, J. Hutter, and J. VandeVondele, “Auxiliary Density Matrix Methods for Hartree-Fock Exchange Calculations," J. Chem. Theory Comput., vol. 6, no. 8, pp. 2348-2364, 2010.

[15] W. Martienssen and H. Warlimont, Springer Handbook of Condensed Matter and Materials Data. 2005

[16] S. B. Zhang and J. E. Northrup, "Chemical potential dependence of defect formation energies in GaAs: Application to Ga self-diffusion," Phys. Rev. Lett., vol. 67, no. 17, pp. 2339-2342, Oct. 1991.

[17] S. Lany and A. Zunger, "Accurate prediction of defect properties in density functional supercell calculations," Model. Simul. Mater. Sci. Eng., vol. 17, no. 8, p. 84002, 2009.

[18] D. E. Eastman, "Photoelectric Work Functions of Transition, Rare-Earth, and Noble Metals," Phys. Rev. B, vol. 2, no. 1, pp. 1-2, Jul. 1970.

[19] N. F. and A. O. and K. M. and S. Miyazaki, "Evaluation of valence band top and electron affinity of $\mathrm{SiO} 2$ and Si-based semiconductors using $\mathrm{X}$ ray photoelectron spectroscopy," Jpn. J. Appl. Phys., vol. 55, no. 8S2, p. 08PC06, 2016.

[20] Y.-C. Yeo, P. Ranade, T.-J. King, and C. Hu, "Effects of high- $\kappa$ gate dielectric materials on metal and silicon gate workfunctions," IEEE Electron Device Lett., vol. 23, no. 6, pp. 342-344, 2002.

[21] A. M. El-Sayed, M. B. Watkins, A. L. Shluger, and V. V. Afanas'Ev, "Identification of intrinsic electron trapping sites in bulk amorphous silica from ab initio calculations," Microelectron. Eng., vol. 109, pp. 68 $71,2013$.

[22] D. Z. Gao, A.-M. El-Sayed, and A. L. Shluger, "A mechanism for Frenkel defect creation in amorphous $\mathrm{SiO}_{2}$ facilitated by electron injection," Nanotechnology, vol. 27, no. 50, p. 505207, 2016.

[23] J. D. Mcbrayer, R. M. Swanson, and T. W. Sigmon, "Diffusion of Metals in Silicon Dioxide," no. June, pp. 1242-1246, 1986.
Aono, "Generic Relevance of Counter Charges for Cation-Based Nanoscale Resistive Switching Memories," ACS Nano, vol. 7, no. 7, pp. 6396-6402, Jul. 2013.

[25] Y. Wimmer, A.-M. El-Sayed, W. Gös, T. Grasser, and A. L. Shluger, "Role of hydrogen in volatile behaviour of defects in $\mathrm{SiO}_{2}$-based electronic devices," Proc. R. Soc. A Math. Phys. Eng. Sci., vol. 472, no. 2190, Jun. 2016. 